\title{
Species Turnover through Time: Colonization and Extinction Dynamics across Metacommunities
}

\author{
Felipe Micali Nuvoloni, ${ }^{1}$ Reinaldo José Fazzio Feres, ${ }^{2}$ and Benjamin Gilbert ${ }^{3, *, \dagger}$
}

1. Programa de Pós Graduação em Biologia Animal, Instituto de Biociências Letras e Ciências Exatas, Universidade Estadual Paulista, 2265 Cristóvão Colombo Street, São José do Rio Preto, Brazil; 2. Departmento de Zoologia e Botânica, Instituto de Biociências Letras e Ciências Exatas, Universidade Estadual Paulista, 2265 Cristóvão Colombo Street, São José do Rio Preto, Brazil; 3. Department of Ecology and Evolutionary Biology, University of Toronto, 25 Harbord Street, Toronto M5S 3G5, Canada

Submitted March 14, 2015; Accepted January 25, 2016; Electronically published April 13, 2016

Online enhancements: appendix, zip file. Dryad data: http://dx.doi.org/10.5061/dryad.4t393.

Ав STRACT: Island biogeography and metacommunity theory often use equilibrium assumptions to predict local diversity, yet nonequilibrium dynamics are common in nature. In nonequilibrium communities, local diversity fluctuates through time as the relative importance of colonization and extinction change. Here, we test the prevalence and causes of nonequilibrium dynamics in metacommunities of mites associated with rubber trees distributed over large spatial $(>1,000 \mathrm{~km})$ and temporal (>30-60 generations) scales in Brazil. We measured colonization and extinction rates to test species turnover and nonequilibrium dynamics over a growing season. Mite metacommunities exhibited nonequilibrium dynamics for most months of the year, and these dynamics tracked climatic conditions. Monthly shifts in temperature of more than $1^{\circ} \mathrm{C}$ resulted in nonequilibrium dynamics, as did mean temperatures outside of two critical ranges. Nonequilibrium dynamics were caused by a change in colonization with temperature change and changes in both colonization and extinction with absolute temperature. Species turnover showed different trends; high relative humidity increased both colonization and extinction rates, increasing turnover but not nonequilibrium dynamics. Our study illustrates that testing nonequilibrium dynamics can provide new insights into the drivers of colonization, extinction, and diversity fluctuations in metacommunities.

Keywords: Acari, metacommunity, patch dynamic, species time relationship, Hevea brasiliensis, succession.

\section{Introduction}

The processes that generate species turnover through time are central to many ecological theories (Rosenzweig 1995, 1998). Island biogeography theory, for example, proposes that diversity on islands is structured through processes that promote or dampen colonization, speciation, and local extinction rates, a premise that has since been adopted by meta-

\footnotetext{
* Corresponding author; e-mail: benjamin.gilbert@utoronto.ca.

+ ORCID: Gilbert, http://orcid.org/0000-0002-4947-6822.

Am. Nat. 2016. Vol. 187, pp. 786-796. @ 2016 by The University of Chicago. 0003-0147/2016/18706-56134\$15.00. All rights reserved. DOI: $10.1086 / 686150$
}

community and neutral theory (MacArthur and Wilson 1967; Hubbell 2001; Leibold et al. 2004). Each of these bodies of theory can be used to predict temporal signatures of diversity over ecological timescales (MacArthur and Wilson 1967; Loreau et al. 2003; Gilbert et al. 2006). Because these temporal patterns of diversity-and the processes that structure them-are amenable to empirical tests, researchers have increased efforts to characterize and quantify species turnover through time (Adler and Lauenroth 2003; Anderson 2007; White et al. 2010).

A central question for understanding patterns of temporal diversity is whether diversity reflects an equilibrium condition of species loss and gain through time. On ecological timescales, where speciation is assumed to be unimportant, equilibrium diversity occurs when colonization and extinction events are equal on average. Although much of our understanding of island biogeography and metacommunities reflects equilibrium assumptions of species gain and loss (e.g., MacArthur and Wilson 1967; Tilman 1994; Klausmeier 2001), nonequilibrium communities may be common and provide novel insights into the processes that structure diversity through time (Hastings 2004). For example, insect diversity often fluctuates seasonally in temperate and tropical regions; determining the ecological drivers of nonequilibrium dynamics is necessary for understanding a large portion of the diversity in these forests (Kishimoto-Yamada and Itioka 2015). Moreover, as chronic shifts in environmental conditions proceed with climate change, understanding nonequilibrium dynamics may be increasingly necessary for species conservation (Wolkovich et al. 2014).

A number of approaches have been developed to model species turnover through time, with the usefulness of different approaches depending on whether diversity is at equilibrium. The species-time relationship is an approach that models average accumulation of diversity over samples separated by different time intervals and is appropriate for modeling the accumulation of diversity in communities that are 
at equilibrium (fig. 1; Adler and Lauenroth 2003). Multivariate measures of turnover, like Sorensen distance, are mathematically related to the slope of a species-time relationship when communities are at equilibrium (Bennett and Gilbert 2015). However, these measures can also be calculated separately over any given time period, allowing researchers to test ecological drivers of turnover in nonequilibrium communities (Korhonen et al. 2010).

Approaches have also been developed to examine colonization and extinction dynamics more directly, allowing researchers to test drivers of temporal change in nonequilibrium communities (Brown et al. 2001; Anderson 2007). For example, Anderson showed that some turnover measures, like Sorensen distance $\left(D_{\text {Sorensen }}\right)$, can be decomposed into their component parts to examine gains (or net coloni- zation $[\mathbf{C}]$ ) and losses (or realized extinctions [E]; Anderson 2007):

$$
D_{\text {Sorensen }}=\frac{(\mathbf{C} / \bar{S})+(\mathbf{E} / \bar{S})}{2}
$$

Here, colonization and extinction events are expressed per species in a patch using mean species richness at both time periods $\bar{S}$; these per species measures are useful when comparing among ecological communities that differ in their species pools or when variation increases with mean species richness, as has been derived for some models and random sampling processes (e.g., MacArthur and Wilson 1967). In the remainder of this article, all measures of colonization and extinction use this per species standardization.
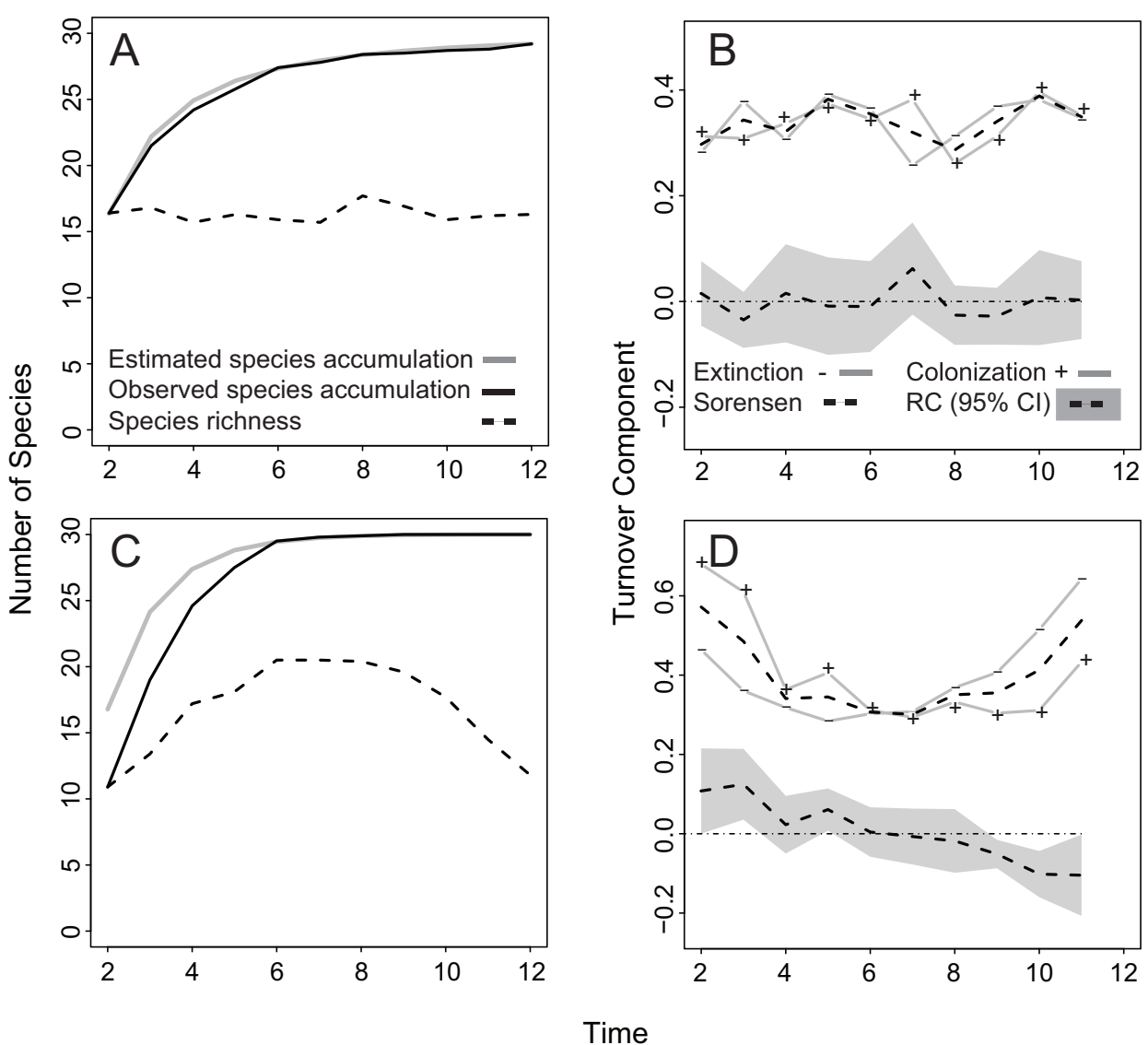

Figure 1: Conceptual model illustrating when species accumulation curves calculated using average (estimated) accumulation differ from observed species accumulation curves and how these differences relate to turnover metrics. $A$, Averaging approach used in species-time relationship studies is valid when species richness is at equilibrium (gray and black line do not differ). $B$, Turnover components calculated from $A$; turnover (Sorensen distance) is the mean of colonization (plus signs) and extinction (minus signs). Relative colonization (RC) is not significantly different from 0 when richness is at equilibrium. $C$, Averaging approach is not valid in nonequilibrium communities, when species richness changes as a result of nonrandom changes in the relative importance of colonization and extinction. $D$, Turnover components calculated from simulations in $C$. Turnover components are calculated using equations (1) and (2). Specific methods and simulation code are given in the appendix, available online. CI, confidence interval. 
Colonization and extinction can be modeled as functions of ecological drivers, such as climatic variation, and can also be compared directly to determine whether a community is in equilibrium. We present a metric to facilitate the latter comparison, which we call relative colonization (RC):

$$
\begin{aligned}
\mathrm{RC} & =\frac{\mathbf{C}}{\bar{S}}-D_{\text {Sorensen }} \\
& =\frac{\mathbf{C}-\mathbf{E}}{2 \bar{S}},
\end{aligned}
$$

which expresses colonization of a patch relative to turnover (eq. [2a]) or, equally, the amount of turnover that is due to colonization compared with extinction (eq. [2b]). The RC metric attains a maximum value equal to Sorensen distance when all turnover results from colonization events. Similarly, it attains negative values when more turnover results from extinction events than colonization events, reaching a minimum of -1 when extinction of all species in a community is not offset by colonization (fig. A1; figs. A1-A7 available online).

Unlike previous approaches that quantify colonization and extinction (e.g., Valone and Brown 1995; Brown et al. 2001; Anderson 2007), the RC metric (eqq. [2]) need not be correlated with turnover. Additionally, in cases where species richness is not changing through time because colonization and extinction rates are the same, the $\mathrm{RC}$ metric will be stable and centered on 0 regardless of the time between sampling events, whereas Sorensen distance under the same conditions will increase toward a maximum of 1 as the time between sampling increases. As a result, a simple test of whether RC differs from 0 is sufficient to determine whether colonization or extinction dynamics predominate in a given time period (i.e., if a metacommunity displays nonequilibrium dynamics; fig. 1).

An additional advantage of isolating the effects of colonization and extinction-and their net effect on diversity via the RC metric-is that it allows researchers to model mechanisms that drive nonequilibrium dynamics in species richness. More concretely, changes in species diversity over time are often modeled as a function of environmental conditions, such as changes in temperature or rainfall. By testing the effect of environmental variables on turnover, its components, and RC separately, it is possible to determine when changes in the environment simply speed up both colonization and extinction dynamics and when they cause changes in one to overwhelm the other.

We test colonization and extinction dynamics in metacommunities of mites associated with rubber trees (Hevea brasiliensis Muell. Arg. Euphorbiaceae) in three provinces in Brazil. These communities comprise a spatially extensive sample $(>1,000 \mathrm{~km})$ that was collected over the course of 5 years, for a total of 13 sites that included 250-400 rubber trees each. Each site functions as a metacommunity, with trees acting as local assemblages with hundreds of leaves that are habitat for the mites. Species passively disperse within and among trees by wind or phoresis over long distances and actively disperse over short distances. Rubber trees are deciduous and have synchronized phenological cycles across regions so that all leaves senesce during the dry season (late July or August) and new leaves emerge in September. This annual cycle corresponds to 30-60 generations, depending on the mite species (Feres et al. 2010; Daud et al. 2012). The combination of seasonal and dispersal dynamics makes the mite system particularly well suited to testing metacommunity processes over time.

Our goals for this study were (1) to determine whether our metacommunities were at equilibrium by examining whether colonization and extinction dynamics were directional over time, (2) to determine whether environmental conditions are predictive of colonization and extinction dynamics, and (3) to test whether environmental conditions that drive turnover differ from those that influence RC. We show that jointly considering extinction, colonization, turnover, and RC clarifies the importance of ecological processes that any single measure of turnover would fail to detect.

\section{Methods}

$$
\text { Study System }
$$

We built a data set from five previous surveys (Demite and Feres 2007; Daud et al. 2010; Nuvoloni 2011; Castro 2012; Daud and Feres 2013). These surveys covered 13 sites from three provinces in Brazil and were sampled between 2004 and 2009. The surveys applied the same methodology of sampling, with collection of leaflets occurring monthly or biweekly for the period of 1 year; we included monthly samples in our data set. A set number of trees were sampled in each site by removing a fixed number of leaves from the tree crown. The number of trees sampled and number of leaves varied among surveys but were consistent within surveys (appendix, available online). We discuss how these differences in sampling were addressed in our statistical methods below.

In total, our data contained more than 150 species of mites and approximately 1 million individuals from a total of $225 \mathrm{~m}^{2}$ of leaf area. Voucher specimens are housed in the Acari collection (DZSJRP; http://www.splink.cria.org.br) of the Laboratório de Acarologia, Departamento de Zoologia e Botânica, Universidade Estadual Paulista, São José do Rio Preto, Brazil. All identification was standardized by F. M. Nuvoloni from voucher specimens.

Climatic data were obtained from local meteorological stations and the authors of surveys. The variables included 
were average temperatures $\left({ }^{\circ} \mathrm{C}\right)$, average relative humidity (\%), rainfall for 30 days before sampling ( $\mathrm{mm})$, and sunlight duration (h; all variables taken monthly). From these data, we created two types of variables that were used in analysis. First, we created change variables, expressed below as $\Delta$ temperature, $\Delta$ relative humidity, and so on. Change variables give the change in each variable from month to month and were calculated as the value in the later month minus the value in the earlier month (e.g., temperature ${ }_{\text {June }}-$ temperature $_{\mathrm{May}}$ ). These variables test the hypothesis that a change in diversity is driven by changes in environmental conditions. We then calculated mean variables by taking the means of each environmental variable for each set of two adjacent months (e.g., mean of September and October, October and November). This was done to test associations with the mean environmental condition experienced during a period of turnover. There were no significant correlations between the mean variables and their associated change variables (largest absolute value of $r=0.08$ ). We included both groups of variables in our statistical analyses, because turnover or colonization could be favored by certain conditions, such as high temperatures, or could respond more strongly to changes in environmental conditions, as is often assumed in multivariate tests of turnover (Gilbert and Bennett 2010). Turnover showed a hump-shaped relationship with one variable ( $\Delta$ temperature; residuals of linear analysis had a clear pattern), and a quadratic term for this (centered) variable was therefore included in the turnover analyses. Similarly, rainfall was log transformed to meet analysis assumptions.

\section{Data Analysis}

September was established as the first phenological month $\left(T_{0}\right)$ in order to standardize all sites by date of leaf emergence. To understand how species vary throughout the year and across sites, we estimated temporal alpha diversity (species richness) and cumulative richness per tree as well as species temporal turnover rates (colonization, extinction, $\mathrm{RC}$, and Sorensen) by considering the change in composition from each month to the following month. Our data exclude changes from time 0 to 1 if there were initially no leaves to sample and similarly exclude the end of the phenological year if a given tree had no leaves to sample. Excluding these data points avoids obvious directionality in colonization and extinction that must occur with habitat creation or destruction and also caused the variation in our estimates to be greatest at the first and last months because of low sample sizes.

Because of differences in sampling effort across surveys (different numbers of trees sampled per time period), we first calculated each index on a per tree basis and then generated mean indices for each site $\times$ month combination by averaging across all trees within sites for a given month.
This approach treats each site as a single metacommunity, and generates one measure per metacommunity per time step. To account for systematic differences among sites, we used mixed models for all analyses, with site considered a random factor. Alpha diversity was calculated as the mean diversity per tree in a given site and month. Observed cumulative richness was calculated as the observed cumulative number of species per tree up to the sampling date given. Estimated or averaged cumulative richness was calculated in two ways. First, the cumulative number of species observed on average for the same number of months but assuming sampling through time is random ( $\mathrm{R}$ software for this and all subsequent analyses; vegan library using function specaccum with the exact method). This first approach is closest to Preston's initial proposal for the species-time relationship. Second, we used a nested approach, where month 1 was the average alpha of all months, month 2 was the average of all species accumulated over a 2-month period (i.e., from month 1 to 2 , month 2 to 3 , month 3 to 4 ), and so on (following Adler and Lauenroth 2003). This nested approach provides 12 data points for 1 month, 11 for 2 months, and so on until there is a single data point for 12 months; we used the mean of each time point for each tree. Colonization and extinction were measured relative to mean species richness (eq. [1]), and their average is Sorensen distance (vegdist function in vegan library). RC was calculated according to equation (2); all metrics have a single value for each transition (i.e., one transition from September to October, another for October to November, and so on), providing one less measure per tree than our original number of observations.

We used two approaches to test whether the mite communities were, on average, in equilibrium. First, we calculated the ratio between estimated (using random sampling assumptions) and observed cumulative richness. By definition, these two measures of cumulative richness need to converge at 12 months, when both are equal to the total number of species observed. If the averaging approach is appropriate, the slope of the ratio of these indices should not be statistically different from 0 , meaning that the relative importance of $\mathbf{C}$ and $\mathbf{E}$ are essentially random through time and thus observed cumulative richness does not diverge from a random expectation. This first test was performed using a linear mixed model (lmer function from lme4 library), with site considered a random factor and both the slope and slope $\times$ site terms tested for significance. The appendix provides more details on how missing data were accounted for in both fully random and nested calculations of cumulative richness.

In our second test of nonequilibrium dynamics, we determined whether the average $\mathrm{RC}$ was significantly different from 0 across sampling periods. The null hypothesis for this test is that communities are at equilibrium. This test was performed using phenological month as the pre- 
dictor (with September equal to 1). Month was treated as a linear or class variable as was appropriate from examinations of the data and residuals, and site was included as a random factor (lme function in nlme library). Confidence intervals were generated using the predictSE.lme function in the AICcmodavg library, with the number of sites used to determine the degrees of freedom.

Following these initial analyses, we tested the influence of environmental covariates on colonization, extinction, RC, and Sorensen distance using linear mixed models (lme function in nlme library). We determined the combination of the mean and change variables that predicted each index as follows. For each index, we first calculated the variables that were significant predictors alone and then included these alone and in all linear combinations to create a set of candidate models. These candidate models were then compared using the corrected Akaike information criterion (AICc) for model selection (aictab function in AICcmodavg library), and the best-fitting model (highest weight) was tested for statistical significance. To meet model assumptions, rain was log transformed, and temperature was included as both a (centered) linear variable and a quadratic term. Data for all analyses presented are deposited in the Dryad Digital Repository: http://dx.doi.org/10.5061/dryad.4t393 (Nuvoloni et al. 2016).

\section{Results}

Species diversity followed broadly similar patterns among sites, with the highest mite diversity observed around month 8 (figs. 2, A2). The patterns of alpha diversity largely reflected mite abundances, with the highest diversity occurring when abundances were high (figs. A3, A4; Spearman's $\rho=0.85$, $P<.001)$. The variance in alpha diversity scaled with the mean, and this ratio did not change over time $(P=.19$; fig. A5).

The mite metacommunities displayed nonequilibrium dynamics over most of the season, with climatic conditions appearing to drive some of these dynamics (figs. 2, 3). As a result, modeling turnover using the average accumulation of diversity was not effective in these communities; it consistently overestimated species accumulation in the first months following leaf-out (figs. 2A, A2, A6). Colonization was highest at the beginning of the season but showed a sharp decline in the third month followed by increases in the fourth and fifth months (fig. 2B). This drop in the third month was matched by a rise in extinction, causing Sorensen turnover to remain fairly constant at the beginning of the season (fig. $2 B$ ). In contrast, the shift from colonizationdominated to extinction-dominated turnover in the third month caused RC to diverge from a simple linear trend (AICc weight of analysis by month was 1 , although linear analysis was still a significantly better fit than an intercept-only model). Months 6-9 had an RC that was not significantly different
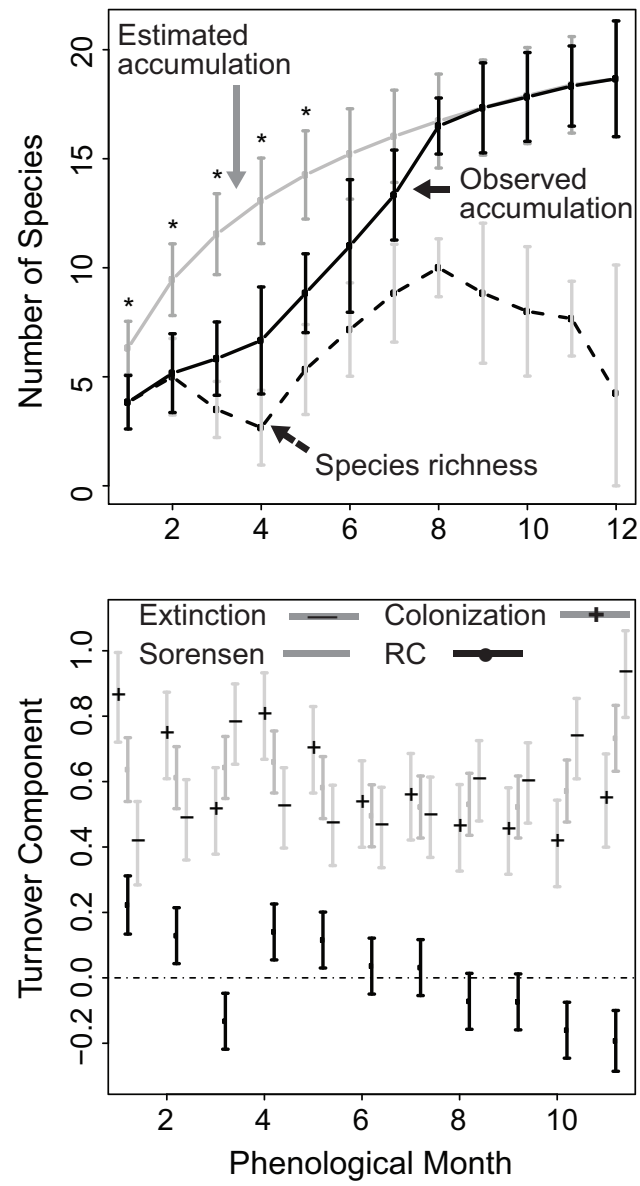

Figure 2: Nonequilibrium dynamics of the mite metacommunities. $A$, Alpha diversity, observed and estimated cumulative richness by phenological month. The selected site (site 13; fig. A2, available online) is closest to the average among sites in terms of the difference between the cumulative richness observed versus estimated using the averaging approach. Asterisks above the data points signify a significant difference between observed and estimated cumulative richness on the basis of $t$ tests with a sequential Bonferroni correction. Results for all sites are shown in figure A6, available online. $B$, Turnover components calculated for all metacommunities. Relative colonization (RC; black) is not significantly different from 0 when richness is at equilibrium. All error bars are $95 \%$ confidence intervals, with estimates in $A$ based on the representative site and in $B$ based on variation among sites after averaging across trees within sites.

from 0 (fig. $2 B$ ), meaning that communities were close to equilibrium species richness in only this subset of 4 months.

Colonization and extinction responded differently to climatic conditions, generating patterns of turnover that were distinct from relative colonization (RC; figs. 3, A7). Absolute temperature, change in temperature, and relative humidity were the climatic variables that were included in the final models for these four responses (fig. 3). Colonization was highest and extinction lowest at approximately $25^{\circ} \mathrm{C}$, causing $\mathrm{RC}$ to peak at this temperature (fig. $3 A, 3 B$ ). A relatively steep 

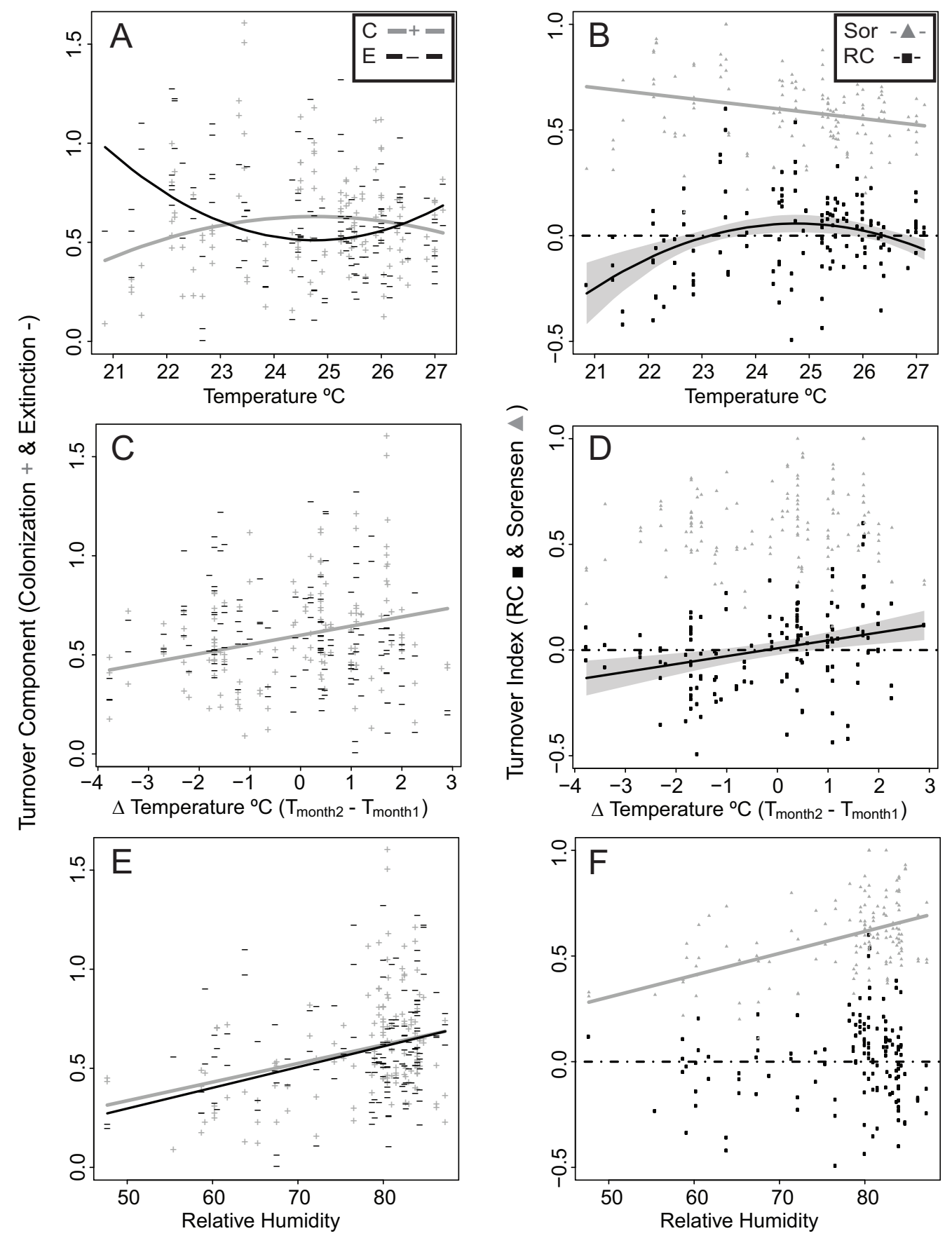

Figure 3: Climatic correlates of colonization and extinction $(A, C, E)$ and turnover metrics: relative colonization (RC) and Sorensen distance $(B, D, F) . A$, Temperature causes opposite trends in colonization (plus signs) and extinction (minus signs). $B$, Temperature effect on RC reflects the nonlinear difference in colonization and extinction patterns, whereas Sorensen decreases on average with higher temperature. $C, D$, Change in temperature between months causes colonization and RC to increase but has no detectable effect on extinction and Sorensen. E, $F$, Relative humidity causes colonization and extinction to increase at almost equal rates, which increases Sorensen but has no detectable effect on RC. In $A-F$, lines are included for a metric only if the explanatory variable was statistically significant. The dashed line at zero $(B, D, F)$ marks equilibrium species richness, as measured by the RC metric. 
rise in extinction at low temperatures caused Sorensen turnover to be greatest at low temperatures (fig. $3 B$ ). Increases in temperature from one month to the next also generated high colonization, while extinction showed a negative but nonsignificant trend (fig. 3C). These dynamics caused RC to increase as temperatures rose between months, whereas Sorensen turnover showed no significant response (fig. 3D). Finally, extinction and colonization increased at almost identical rates with relative humidity (fig. $3 E$ ), causing an increase in Sorensen turnover and no change in RC (fig. $3 F$ ).

The confidence intervals for $\mathrm{RC}$ give an indication of the climatic conditions that allow communities to maintain an equilibrium number of species. In particular, RC was not significantly different from 0 when mean temperature was moderately low $\left(22.5^{\circ}-23.5^{\circ} \mathrm{C}\right)$ or high $\left(26^{\circ}-26.5^{\circ} \mathrm{C}\right)$; species richness decreased in more extreme conditions and increased between these temperature windows (fig. 3B). Similarly, consistency in temperature appears necessary to maintain equilibrium conditions; RC was not different from 0 when mean temperature was constant over time (approximately $\pm 1{ }^{\circ} \mathrm{C}$ from one month to the next; fig. $\left.3 D\right)$. These two measures of temperature were independent $(r=0.045)$ and were both present in the final model for $\mathrm{RC}$, indicating that both mean temperature and change in temperature are important for nonequilibrium dynamics.

\section{Discussion}

\section{Mite Metacommunities: Temporal Turnover and Nonequilibrium Dynamics}

Colonization and extinction dynamics in the mite metacommunities changed at different rates over time, suggesting that local diversity is rarely at equilibrium in this system (fig. 2). These temporal patterns in metacommunity dynamics resulted in changing rates of species turnover throughout the growing season, a phenomenon that is likely found in many ecosystems (e.g., Korhonen et al. 2010). Using the RC metric to decompose turnover, we were able to determine when temporal changes caused nonequilibrium metacommunity dynamics and the proximate climatic drivers of these dynamics. These insights provide a novel understanding of species turnover through time and how it can inform our understanding of colonization and extinction dynamics in metacommunities.

Many authors have proposed that metacommunity dynamics underlie both species-area and species-time relationships through both extinction-colonization dynamics and sampling of environmental heterogeneity (e.g., Rosenzweig 1998; Rosenzweig and Ziv 1999; Adler et al. 2005; Scheiner et al. 2011). Metacommunities offer a clear example of why the effects of space and time on diversity are often impossible to separate; spatial turnover among similar habitat patches results from species changing over time through stochastic colonization and extinction events. Similarly, temporal turnover requires that species have refuges in order to persist over the long term; these refuges may often be spatial (e.g., Venable and Brown 1988; Loreau et al. 2003). Our study suggests that using time series to model the components of turnover provides a more mechanistic understanding of the independent and combined effects of colonization and extinction on diversity and may also lead to an understanding of the factors that drive these processes.

Applying our approach to natural metacommunities allowed us to identify distinct effects of environmental drivers on species turnover and nonequilibrium dynamics that highlight the importance of isolating temporal metacommunity processes. For example, if species turnover were considered alone, it would appear that diversity was most influenced by low temperatures and high relative humidity (fig. $3 B$, $3 F)$. Although turnover was highest at low temperatures, extinction exceeded colonization in these conditions, resulting in a decrease in local richness (fig. $3 A, 3 B$ ). In contrast, high relative humidity increased both colonization and extinction rates and therefore caused high turnover but was not a significant predictor of nonequilibrium dynamics (fig. $3 E, 3 F$ ).

The impact of environmental change on temporal diversity reflects its influence on the net colonization and extinction rates of all species and, as a result, may be predictable from the population ecology of constituent species. Although data is lacking for most mite species, our results are consistent with the biology of mite species that have been well studied. For example, relative humidity increases egg hatching rates of six common predatory mite species on rubber trees, with relative humidity above $70 \%$ causing the highest rates of hatching and below 50\% causing hatching failure in some species (De Vis et al. 2006). Higher hatching rates should increase colonization for the predatory mites, which may in turn lower population sizes and increase the risk of extinction in their prey species. However, relative humidity is also a strong predictor of mite infection by the fungal pathogen Hirsutella thompsonii (Nuvoloni et al. 2014). The Hirsutella genus produces many of the most important pathogens of eriophyoid mites and likely represents an important cause of mortality and extinction in the mite community (Nuvoloni et al. 2014). Given the opposing effects of relative humidity on community dynamics through increased hatching, predation, and pathogen infection, it is not surprising to find that it increases species turnover but not RC.

The effects of temperature and seasonal changes in temperature are also consistent with the biology of well-studied mites and general metabolic processes. For many organisms, reproductive rates increase exponentially with temperature to a maximum, at which point they quickly decline (Savage et al. 2004; Dell et al. 2011; Amarasekare and Savage 2012). 
Although the temperature dependence of Neotropical mites is not well studied, the data available indicate that the highest net reproductive rates and finite rates of increase occur from $25^{\circ}$ to $30^{\circ} \mathrm{C}$ (Childers et al. 1991; Gerson 1992; Ali 1998). This peak in reproductive rates is slightly higher than the mean temperatures that lead to the highest level of relative colonization (fig. $3 B$ ), a difference that is expected because of the nonlinearity of the temperature-fitness relationship (Vasseur et al. 2014). Importantly, high reproductive rates are expected to reduce the probability of stochastic extinction events when populations are initially rare, leading to higher colonization and lower extinction rates (Lande et al. 2003).

Temperature change between contiguous months was also important for metacommunity dynamics, with large increases in temperature increasing colonization (fig. $3 C$ ). This effect of changing temperature can arise when interacting species have different temperature sensitivities, which can induce both transient and long-term shifts in species dynamics (Gilbert et al. 2014). The importance of changing temperatures on mites reflects the influence that variation in environmental conditions, rather than mean environmental conditions, may have on metacommunity processes; nonequilibrium dynamics were observed when absolute changes in mean temperature between months exceeded $1^{\circ} \mathrm{C}$ (fig. $3 D$ ). Surprisingly, changes in environmental conditions were not predictive of species turnover. Many spatial analyses of turnover test the effects of environmental change, not the ambient environment per se, and thus may miss important drivers of metacommunity dynamics (Gilbert and Bennett 2010). Indeed, because only temporal data is suited to decoupling colonization and extinction from turnover, an important question for future studies is whether the different effects of ambient versus changing environments that we observed hold across ecosystems.

Temporal changes in diversity are likely due not only to external environmental conditions, but also to changes in habitat quality - the rubber tree leaf in the case of mites. Rubber tree leaves are partially defended by the release of hydrogen cyanide when damaged, but the amount of hydrogen cyanide released changes with leaf age (Lieberei 1988, 2007; Kadow et al. 2012). Similarly, leaf maturation alters leaf photosynthetic properties and associated chemical composition, such as the amount of soluble proteins and the presence of lignin (Lieberei 2007; Miguel et al. 2007). These changes are expected to favor some species while disadvantaging others, causing predictable shifts in species composition (Lieberei 2007; Daud et al. 2012; Kadow et al. 2012). In our system, full leaf maturation occurs after approximately 50 days in natural field conditions (Miguel et al. 2007); species changes associated with leaf maturation would be captured in our months 2 and 3 measurements, which is when we observe the first shift toward large numbers of extinctions. Interestingly, a previous meta-analysis of arthropod successional dynamics showed no change in extinction rates over time, reflecting either differences among systems or the short time frames of many arthropod studies (Anderson 2007). More generally, our results suggest that deterministic shifts in habitat quality with habitat age likely influence mite temporal diversity, just as they have been shown to be important to a variety of other ecosystems (e.g., Nilsson and Rosenberg 2000; SteffanDewenter and Tscharntke 2002).

The temporal dynamics in our metacommunities are distinct from those in classic metacommunity paradigms, which focus on spatial determinants of diversity, yet clear analogs to some of the spatial determinants can be identified (Chesson 2003; Abrams et al. 2013). For example, the species sorting paradigm is simply niche partitioning driven by spatial environmental heterogeneity (Leibold et al. 2004); this spatial partitioning allows species to coexist if they differ sufficiently in how they partition the environment and have some restriction to dispersal (Gilbert 2012). Environmental partitioning through time can occur via the storage effect (Chesson 1994, 2003) and is only slightly more restrictive than the spatial partitioning required for species sorting (Abrams et al. 2013). Such mechanisms can drive shifts in abundance and diversity with environmental fluctuations (Angert et al. 2009; Jones and Gilbert 2016). Our results raise the possibility that these temporal mechanisms may be important determinants of diversity in metacommunities and lead to nonequilibrium colonization and extinction dynamics that are rarely captured in purely spatial models. Although hypotheses such as the storage effect require more detailed tests, their potential importance suggests that further study is warranted.

The metacommunity patterns that we observed also highlight other questions for future research, such as the role of environmental synchrony in maintaining diversity. Most previous research has predicted that environmental fluctuations destabilize metapopulations and metacommunities because of either correlated fluctuations in population size across patches (Gaggiotti and Hanski 2004) or the inability of species to persist during periods of unsuitable conditions (Loreau et al. 2003). The temporal patterns of colonization and extinction that we observed were surprisingly consistent, considering that the metacommunities were distributed over hundreds of kilometers and sampled in separate years (fig. 2). This consistency across our study sites appeared to result from seasonal climatic variation (fig. 3) and is likely common in many ecosystems that experience predictable temporal changes, be it seasonally or over longer periods, as is typical of multiyear climatic oscillations (e.g., Wright and Calderón 2006; Kane 2011). An important goal for these systems is to identify when synchrony among sites destabilizes diversity and when it functions opposite to classic predictions by stabilizing metacommunity dynamics (Vasseur and Fox 2007). 


\section{Comparing Turnover and Nonequilibrium Dynamics among Metacommunities}

The mathematical relationships among RC, Sorensen distance, colonization, and extinction raise the question of when these metrics are necessary (eqq. [1], [2]; fig. A7). Previous research has highlighted the value of Sorensen distance-and several other turnover metrics-as a standardized measure of beta diversity and variation among communities, despite its relationship to colonization and extinction (eq. [1]; Anderson 2007; Legendre and De Caceres 2013; Bennett and Gilbert 2015). We argue that RC can play a similar role in understanding nonequilibrium dynamics. In the simplest case, RC provides a paired contrast that can be used to test for nonequilibrium dynamics (fig. $2 B$ ). Its greatest value, however, is as a standardized metric for comparing the direction and strength of nonequilibrium dynamics among communities that may differ greatly in average diversity (e.g., site 13 vs. site 12; fig. A2). This standardization allows for tests of common causal mechanisms that structure nonequilibrium dynamics (fig. 3), which are becoming increasingly important as climate change alters the nature of temporal fluctuations in climatic conditions (Wolkovich et al. 2014).

It is important to note that although our study examines changes to local communities (i.e., mites on individual trees) within metacommunities (forests or plantations), RC could be applied more broadly to understand changes to species persistence. For example, just as we examine turnover and RC within each tree, an exhaustively sampled metacommunity would provide data on when species are gained or lost from the metacommunity as a whole. Although infeasible in the mite metacommunities studied, exhaustive sampling would allow for tests of metapopulation extinctions, which are one potential end point of nonequilibrium dynamics driven by species responses to environmental fluctuations (Lande et al. 2003; Loreau et al. 2003). Similarly, RC could clarify how local diversity responds to regional diversity following species invasions. In both cases, the RC approach could provide a measure of nonequilibrium dynamics that links temporal processes at local scales to the diversity of the entire metacommunity.

The RC index that we have presented may also be used to understand other ecological patterns, such as the scale dependence of SARs. The shape and slope of SARs is often considered to represent different ecological dynamics: exponential SARs result from sampling processes, power function SARs result from sampling multiple habitats or areas with distinct evolutionary histories, and logistic SARs result from exhaustive sampling of a bioregion (Rosenzweig 1995; He and Legendre 1996). The first of these-the sampling effectresults from the finite number of individuals in small samples and constrains the number of species that may be present and result in higher turnover (He and Legendre 1996; Fridley et al. 2006). The sampling effect is relevant to metacommunity dy- namics; it measures the rate of species turnover among ecologically equivalent patches. A sampling effect also occurs over time, and this small-scale temporal turnover has even been used to account for a spatial sampling effect (Fridley et al. 2006). Contrasting RC with turnover allows us to isolate this sampling effect because random colonization and extinction events cancel out of the RC metric (eq. [2b]) but contribute to turnover metrics like Sorensen distance.

The interplay of ecological and evolutionary processes that structure species diversity on islands may also be better understood using RC or a similar metric aimed at population dynamics. Many expansions of island biogeography theory have incorporated speciation rates into temporal changes in diversity, especially on islands where these rates are expected to match or exceed colonization rates (e.g., Rosenzweig and Ziv 1999; Gillespie and Baldwin 2009). Incorporating ecological dynamics of colonization and extinction into evolutionary processes - such as gene flow, population divergence, and admixture-is increasingly providing insights into island diversity (Gillespie 2016). Our approach to detecting nonequilibrium dynamics may offer new insights into when ecological dynamics provide opportunities for the emergence of new species and, when considered at a population level, for when periods of high gene flow or isolation are likely to occur. Such considerations of nonequilibrium fluctuations in species diversity and local abundances are a promising avenue for future research in ecology and evolutionary biology.

\section{Acknowledgments}

We thank R. D. Daud, E. B. de Castro, P. R. Demite, F. A. Hernandes, and E. R. Silva for providing access to the data sets from publications, theses, and dissertations. The Fundação de Amparo à Pesquisa do Estado de São Paulo provided fellowships to F.M.N. (2010/19935-1, 2013/09600-0), Conselho Nacional de Desenvolvimento Científico e Tecnológico provided research support and funding to R.J.F.F. (303435/2013-5), Plantações Michelin da Bahia and Plantações E. Michelin supported the field work, and the Natural Sciences and Engineering Research Council of Canada provided research funding to B.G.

\section{Literature Cited}

Abrams, P. A., C. M. Tucker, and B. Gilbert. 2013. Evolution of the storage effect. Evolution 67:315-327.

Adler, P. B., and W. K. Lauenroth. 2003. The power of time: spatiotemporal scaling of species diversity. Ecology Letters 6:749-756.

Adler, P. B., E. P. White, W. K. Lauenroth, D. M. Kaufman, A. Rassweiler, and J. A. Rusak. 2005. Evidence for a general species-timearea relationship. Ecology 86:2032-2039.

Ali, F. 1998. Life tables of Phytoseiulus macropilis (Banks) (Gamasida: Phytoseiidae) at different temperatures. Experimental and Applied Acarology 22:335-342. 
Amarasekare, P., and V. Savage. 2012. A framework for elucidating the temperature dependence of fitness. American Naturalist 179: 178-191.

Anderson, K. 2007. Temporal patterns in rates of community change during succession. American Naturalist 169:780-793.

Angert, A. L., T. E. Huxman, P. Chesson, and D. L. Venable. 2009. Functional tradeoffs determine species coexistence via the storage effect. Proceedings of the National Academv of Sciences of the USA 106:11641-11645.

Bennett, J. R., and B. Gilbert. 2015. Contrasting beta diversity among regions: how do classical and multivariate approaches compare? Global Ecology and Biogeography 25:368-377. doi:10.1111/geb.12413.

Brown, J. H., S. K. M. Ernest, J. M. Parody, and J. P. Haskell. 2001. Regulation of diversity: maintenance of species richness in changing environments. Oecologia (Berlin) 126:321-332.

Castro, E. B. 2012. Diversidade e sazonalidade da comunidade de ácaros em três clones de seringueira no sudeste do Estado da Bahia. MSc thesis. Universidade Estadual Paulista, São José do Rio Preto, Brazil.

Chesson, P. 1994. Multispecies competition in variable environments. Theoretical Population Biology 45:227-276.

- 2003. Quantifying and testing coexistence mechanisms arising from recruitment fluctuations. Theoretical Population Biology 64:345-357.

Childers, C. C., M. M. Abou-Setta, and M. S. Nawar. 1991. Biology of Eutetranychus banksi: life tables on "marsh" grapefruit leaves at different temperatures (Acari: Tetranychidae). International Journal of Acarology 17:29-35.

Daud, R. D., E. R. O. da Silva, and R. J. F. Feres. 2010. Influência de Cecropia pachystachya na incidência de ácaros (Arachnida, Acari) e de Leptopharsa heveae (Insecta, Hemiptera) em cultivo de seringueira. Revista de Biologia Neotropical 7:49-60.

Daud, R. D., and R. J. F. Feres. 2013. Community structure of mites (Arachnida: Acari) in six rubber tree clones. International Journal of Acarology 39:589-596.

Daud, R. D., R. J. F. Feres, and F. A. Hernandes. 2012. Seasonal suitability of three rubber tree clones to Calacarus heveae (Acari, Eriophyidae). Experimental and Applied Acarology 56:57-68.

Dell, A. I., S. Pawar, and V. M. Savage. 2011. Systematic variation in the temperature dependence of physiological and ecological traits. Proceedings of the National Academv of Sciences of the USA 108:10591-10596.

Demite, P. R., and R. J. F. Feres. 2007. Ocorrência e flutuação populacional de ácaros associados a seringais vizinhos de fragmentos de Cerrado. Neotropical Entomology 36:117-112.

De Vis, R. M. J., G. J. Moraes, and M. R. Bellini. 2006. Effect of air humidity on the egg viability of predatory mites (Acari: Phytoseiidae, Stigmaeidae) common on rubber trees in Brazil. Experimental and Applied Acarology 38:25-32.

Feres, R. J. F., M. Del'Arco, and R. D. Daud. 2010. Biological cycle of Tenuipalpus heveae Baker (Acari, Tenuipalpidae) on leaflets of three rubber tree clones. Revista Brasileira de Entomologia 54:298-303.

Fridley, J. D., R. K. Peet, E. van der Maarel, and J. H. Willems. 2006. Integration of local and regional species-area relationships from spacetime species accumulation. American Naturalist 168:133-143.

Gaggiotti, O. E., and I. Hanski. 2004. Mechanisms of population extinction. Pages 337-366 in I. Hanski and O. E. Gaggiotti, eds. Ecology, genetics and evolution of metapopulations. Elsevier, Amsterdam.

Gerson, U. 1992. Biology and control of the broad mite, Polyphagotarsonemus latus (Banks) (Acari: Tarsonemidae). Experimental and Applied Acarology 13:163-178.
Gilbert, B. 2012. Joint consequences of dispersal and niche overlap on local diversity and resource use. Journal of Ecology 100:287-296.

Gilbert, B., and J. R. Bennett. 2010. Partitioning variation in ecological communities: do the numbers add up? Journal of Applied Ecology 47:1071-1082.

Gilbert, B., W. F. Laurance, E. G. Leigh, and H. E. M. Nascimento. 2006. Can neutral theory predict the responses of Amazonian tree communities to forest fragmentation? American Naturalist 168:304-317.

Gilbert, B., T. D. Tunney, K. S. McCann, J. P. DeLong, D. A. Vasseur, V. Savage, J. B. Shurin, et al. 2014. A bioenergetic framework for the temperature dependence of trophic interactions. Ecology Letters 17:902-914.

Gillespie, R. G. 2016. Island time and the interplay between ecology and evolution in species diversification. Ecological Applications 9: 53-73.

Gillespie, R. G., and B. G. Baldwin. 2009. Island biogeography of remote archipelagos: interplay between ecological and evolutionary processes. Pages 358-387 in J. Losos and R. Ricklefs, eds. The theory of island biogeography revisited. Princeton University Press, Princeton, NJ.

Hastings, A. 2004. Transients: the key to long-term ecological understanding? Trends in Ecology and Evolution 19:39-45.

He, F., and P. Legendre. 1996. On species-area relations. American Naturalist 4:719-737.

Hubbell, S. P. 2001. The unified neutral theory of biodiversity and biogeography. Princeton University Press, Princeton, NJ.

Jones, N. T., and B. Gilbert. 2016. Changing climate cues differentially alter zooplankton dormancy dynamics across latitudes. Iournal of Animal Ecology 85:559-569. doi:10.1111/1365-2656.12474.

Kadow, D., K. Voß, D. Selmar, and R. Lieberei. 2012. The cyanogenic syndrome in rubber tree Hevea brasiliensis: tissue-damagedependent activation of linamarase and hydroxynitrile lyase accelerates hydrogen cyanide release. Annals of Botanv 109:1253-1262.

Kane, J. 2011. Multiyear variability of phytoplankton abundance in the Gulf of Maine. ICES Journal of Marine Science 68:1833-1841.

Kishimoto-Yamada, K., and T. Itioka. 2015. How much have we learned about seasonality in tropical insect abundance since Wolda (1988)? Entomological Science 18:407-419.

Klausmeier, C. A. 2001. Habitat destruction and extinction in competitive and mutualistic metacommunities. Ecology Letters 4:57-63.

Korhonen, J. J., J. Soininen, and H. Hillebrand. 2010. A quantitative analysis of temporal turnover in aquatic species assemblages across ecosystems. Ecology 91:508-517.

Lande, R., S. Engen, and B.-E. Sæther. 2003. Stochastic population dynamics in ecology and conservation. Oxford University Press, Oxford.

Legendre, P., and M. De Cáceres. 2013. Beta diversity as the variance of community data: dissimilarity coefficients and partitioning. Ecology Letters 16:951-963.

Leibold, M. A., M. Holyoak, N. Mouquet, P. Amarasekare, J. M. Chase, M. F. Hoopes, R. D. Holt, et al. 2004. The metacommunity concept: a framework for multi-scale community ecology. Ecology Letters 7:601-613.

Lieberei, R. 1988. Relationship of cyanogenic capacity (HCN-c) of the rubber tree Hevea brasiliensis to susceptibility to Microcylus ulei, the agent causing South American leaf blight. Journal of Phytopathology 122:54-67.

- 2007. South American leaf blight of the rubber tree (Hevea spp.): new steps in plant domestication using physiological features and molecular markers. Annals of Botany 100:1125-1142. 
Loreau, M., N. Mouquet, and A. Gonzalez. 2003. Biodiversity as spatial insurance in heterogeneous landscapes. Proceedings of the National Academy of Sciences of the USA 100:12765-12770.

MacArthur, R. A., and E. O. Wilson. 1967. The theory of island biogeography. Princeton University Press, Princeton, NJ.

Miguel, A. A., L. E. M. de Oliveira, P. A. R. Cairo, and D. M. de Oliveira. 2007. Photosynthetic behavior during the leaf ontogeny of rubber tree clones [Hevea brasiliensis (Wild. ex. Adr. de Juss) Muell. Arg.], in Lavras MG. Ciência e Agrotecnologia 31:91-97.

Nilsson, H. C., and R. Rosenberg. 2000. Succession in marine benthic habitats and fauna in response to oxygen deficiency: analysed by sediment profile-imaging and by grab samples. Marine Ecology Progress Series 197:139-149.

Nuvoloni, F. M. 2011. Influência da vegetação nativa de Mata Atlântica sobre a ocorrência e distribuição de ácaros (Acari) da seringueira (Hevea brasiliensis Muell Arg.) no estado da Bahia. MSc thesis. Universidade Estadual Paulista, São José do Rio Preto, Brazil.

Nuvoloni, F. M., E. B. de Castro, and R. J. F. Feres. 2014. The role of native vegetation on infection rates of Calacarus heveae (Acari: Eriophyidae) by Hirsutella thompsonii (Ascomycota: Ophiocordycipitaceae). Experimental and Applied Acarology 63:157-169.

Nuvoloni, F. M., R. J. F. Feres, and B. Gilbert. 2016. Data from: Species turnover through time: colonization and extinction dynamics across metacommunities. American Naturalist, Dryad Digital Repository, http://dx.doi.org/10.5061/dryad.4t393.

Rosenzweig, M. L. 1995. Species diversity in space and time. Cambridge University Press, New York.

- 1998. Preston's ergodic conjecture: the accumulation of species in space and time. Pages 311-348 in M. L. Mckinney and J. A. Drake, eds. Biodiversity dynamics: turnover of populations, taxa and communities. Columbia University Press, New York.

Rosenzweig, M. L., and Y. Ziv. 1999. The echo pattern of species diversity: pattern and processes. Ecography 22:614-628.

Savage, V. M., J. F. Gilloly, J. H. Brown, and E. L. Charnov. 2004. Effects of body size and temperature on population growth. American Naturalist 163:429-441.
Scheiner, S. M., A. Chiarucci, G. A. Fox, M. R. Helmus, D. J. McGlinn, and M. R. Willig. 2011. The underpinnings of the relationship of species richness with space and time. Ecological Monographs 81: 195-213.

Steffan-Dewenter, I., and T. Tscharntke. 2002. Insect communities and biotic interactions on fragmented calcareous grasslands-a mini review. Biological Conservation 10:275-284.

Tilman, D. 1994. Competition and biodiversity in spatially structured habitats. Ecology 75:2-16.

Valone, T., and J. Brown. 1995. Effects of competition, colonization, and extinction on rodent species diversity. Science 267:880883.

Vasseur, D. A., J. P. DeLong, B. Gilbert, H. S. Greig, C. D. G. Harley, K. S. McCann, V. Savage, T. D. Tunney, and M. I. O’Connor. 2014. Increased temperature variation poses a greater risk to species than climate warming. Proceedings of the Roval Society B: Biological Sciences 281:20132612. doi:10.1098/rspb.2013.2612.

Vasseur, D. A., and J. W. Fox. 2007. Environmental fluctuations can stabilize food web dynamics by increasing synchrony. Ecology Letters 10:1066-1074.

Venable, D. L., and J. S. Brown. 1988. The selective interactions of dispersal, dormancy and seed size as adaptations for reducing risk in variable environments. American Naturalist 131:360-384.

White, E. P., S. K. M. Ernest, P. B. Adler, A. H. Hurlbert, and S. K. Lyons. 2010. Integrating spatial and temporal approaches to understanding species richness. Philosophical Transactions of the Roval Society B: Biological Sciences 365:3633-3643.

Wolkovich, E. M., B. I. Cook, K. K. McLauchlan, and T. J. Davies. 2014. Temporal ecology in the Anthropocene. Ecology Letters 17: 1365-1379.

Wright, S. J., and O. Calderón. 2006. Seasonal, El Niño and longer term changes in flower and seed production in a moist tropical forest. Ecology Letters 9:35-44.

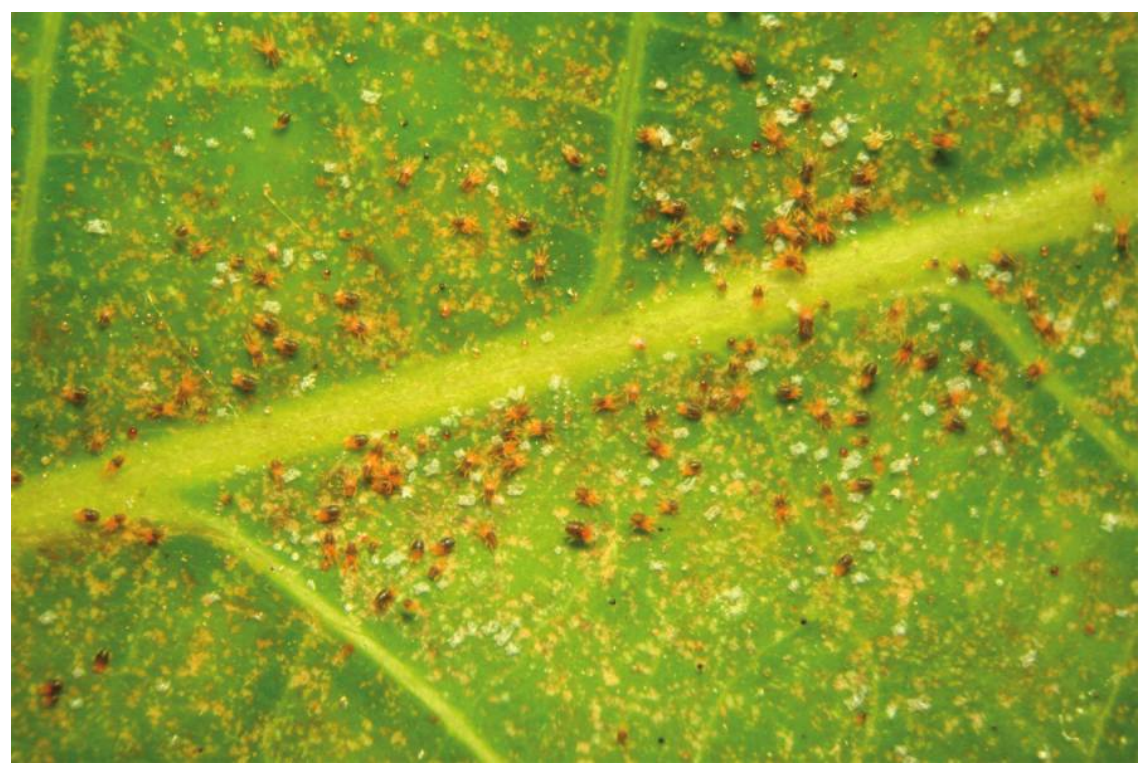

Mites (Oligonychus ilicis) on rubber tree leaflet (Hevea brasiliensis). Photo credit: Felipe Micali Nuvoloni. 